\title{
MOTIVOS PARA ADESÃO AO TRATAMENTO MEDICAMENTOSO DA HIPERTENSÃO ARTERIAL
}

\author{
REASONS FOR ADHERENCE TO DRUG \\ TREATMENT OF HYPERTENSION
}

\author{
Maria de Fátima Mantovani' ${ }^{1}$ Ângela Taís Mattei ${ }^{2}$, Evelyn Nunes da Rocha ${ }^{3}$, \\ Carina Bortolato Major ${ }^{2}$, Elis Martins Ulbrich ${ }^{4}$, Karin Cristina Barboza ${ }^{3}$
}

\begin{abstract}
RESUMO
Objetivo: identificar os aspectos relatados pelas pessoas com hipertensão arterial para aderir ao tratamento medicamentoso. Metodologia: pesquisa descritiva de abordagem quantitativa, realizada em um distrito sanitário do município de Curitiba, Paraná. Os participantes foram 146 adultos hipertensos, com idade entre 18 e 60 anos, considerados aderentes ao tratamento medicamentoso. A coleta de dados foi realizada no domicílio dos participantes, no período de maio de 2013 à fevereiro de 2015, mediante uma entrevista semiestruturada e de um instrumento traduzido e publicado em português o Brief Medication Questionnaire. Os dados foram tabulados em uma planilha do Microsoft Exce ${ }^{\circledR}$ e analisados pelo SPSS versão 20.0. Resultados: alguns motivos para adesão à terapêutica foram a ausência de dificuldades encontradas com a dispensação da medicação e problemas causados pelas medicações anti-hipertensivas, os indivíduos lembrarem de fazer uso das medicações todos os dias e acreditarem que estas funcionam bem. Conclusões: os aspectos relatados pelos participantes que favorecem a adesão ao tratamento foram percebidos mediante a utilização da escala e por meio destes podem-se propor ações para a continuidade desse comportamento.
\end{abstract}

Descritores: Hipertensão; Saúde do Adulto; Adesão à Medicação.
${ }^{1}$ Doutora em Enfermagem pela Universidade de São Paulo (USP), São Paulo, SP, Brasil.

${ }^{2}$ Doutoranda em Enfermagem pela Universidade Federal do Paraná (UFPR), Curitiba, PR, Brasil.

${ }^{3}$ Graduanda em Enfermagem na Universidade Federal do Paraná (UFPR), Curitiba, PR, Brasil.

${ }^{4}$ Doutora em Enfermagem pela Universidade Federal do Paraná (UFPR), Curitiba, PR, Brasil.

\begin{abstract}
Objective: To identify the aspects reported by people with hypertension to adhere to drug treatment. Methodology: descriptive quantitative approach, performed in a health district of the city of Curitiba, State of Parana. Participants were 146 hypertensive adults, aged 18 and 60, considered adherent to medication treatment. Data collection was carried out at the homes of participants, from May 2013 to February 2015, through a semi-structured interview and an instrument translated and published in Portuguese the Brief Medication Questionnaire. Data were tabulated in a Microsoft Exce ${ }^{\circledR}$ spreadsheet and analyzed using SPSS version 20.0. Results: some reasons for adherence were no difficulties with the dispensation of medication and problems caused by antihypertensive medications, individuals remember to make use of medications every day and believe that these work well. Conclusions: aspects reported by the participants that favor adherence to treatment were perceived by using the scale and through these actions may be proposed to continue this behavior.
\end{abstract}

Descriptors: Hypertension; Adult Health; Medication Adherence. 


\section{Introdução}

As doenças cardiovasculares foram a principal causa de morte entre homens e mulheres brasileiras em 2012, representando $26 \%$ e $31 \%$ respectivamente. Entre essas doenças a Hipertensão Arterial Sistêmica (HAS) é a causa mais frequente de morbimortalidade entre a população adulta e idosa. É uma doença silenciosa e agressiva que depende da colaboração e participação ativa do paciente para realizar o controle e a adesão às medidas terapêuticas propostas pela equipe de saúde, a qual é um aspecto fundamental para que haja redução das consequências acarretadas por ela. ${ }^{1-2}$

A HAS possui etiologia multifatorial e destacam-se como fatores predisponentes e inerentes ao estilo de vida, o consumo exacerbado de sal e gorduras, obesidade, estresse, tabagismo, alcoolismo, sedentarismo e o uso de anticoncepcionais. $O$ seu tratamento requer o uso contínuo de medicamentos e mudanças no estilo de vida, desta forma a adesão aos cuidados propostos é fator importante para que os níveis pressóricos sejam controlados a fim de evitar ou minimizar o desenvolvimento de complicações ${ }^{3-4}$.

A adesão ao tratamento refere-se ao nível de cumprimento das medidas terapêuticas propostas pela equipe de saúde, sejam elas medicamentosas ou recomendações para mudanças no estilo de vida ${ }^{5}$. Essa é descrita como a total interação entre fatores sociais, aqueles relativos ao paciente e à equipe de saúde, além das condições socioeconômicas, sexo, medicamentos prescritos e suas respectivas quantidades e efeitos colaterais ${ }^{6-7}$.

Quanto aos fatores envolvidos na adesão, são eles de cunho terapêutico e educacional, destacando-se o conhecimento sobre a doença, a percepção dos fatores de risco, desenvolvimento da consciência para a autogestão e a aceitação da doença. As ações dos profissionais de saúde com vistas à melhora da adesão estão relacionadas à valorização do indivíduo de forma singular e de seus conhecimentos prévios, considerando suas crenças e estilo de vida 4 .

Um dos principais problemas enfrentados pela equipe de saúde é a não adesão ao tratamento, por envolver diversos fatores da vida dos indivíduos, como: dificuldades financeiras, número de medicações prescritas, dificuldade ao acesso no sistema público de saúde, efeitos colaterais dos medicamentos, a cronicidade da doença e a ausência de sintomas. Os baixos índices de adesão ainda prevalecem na população com hipertensão, culminando anualmente no óbito de 400 mil indivíduos brasileiros nos últimos anos ${ }^{8-10}$.

Para que a equipe de saúde possa prestar cuidado à pessoa com hipertensão, tendo em vista a melhoria da aderência ao tratamento como forma de minimizar as complicações, é importante que essa conheça os fatores que facilitam a adesão dos pacientes, utilizando-os como foco na orientação e educação em saúde. Destarte, o objetivo desta pesquisa foi identificar os aspectos relatados pelas pessoas com hipertensão arterial para aderir ao tratamento medicamentoso.

\section{Metodologia}

Trata-se de uma pesquisa descritiva de natureza quantitativa, com dados de um projeto maior intitulado "Construção e Validação de instrumento para o cuidado da hipertensão arterial sistêmica e as representações de cuidado de adultos hipertensos", realizada no período de maio de 2013 à fevereiro de 2015 com 387 pacientes hipertensos de 18 unidades de Saúde de um Distrito Sanitário do município de Curitiba, Paraná. Destes, 146 foram considerados aderentes pela pontuação do questionário de adesão ao tratamento medicamentoso e compõem a amostra deste estudo.

Os critérios para inclusão foram: adultos com 18 a 60 anos, cadastrados e ativos no Programa de Hipertensos e Diabéticos e alcançar a pontuação mínima no Mini Exame do Estado Mental, 13 pontos para analfabetos, 18 para baixa e média escolaridade e 26 para alta. Sendo que, baixa e média escolaridade são aqueles que possuem até oito anos incompletos e acima disso considerou-se como alta escolaridade ${ }^{11}$.

A coleta de dados ocorreu mediante uma entrevista semiestruturada com dados clínicos e sócio demográficos auto relatados e de um instrumento de adesão traduzido e publicado em português o Brief Medication Questionaire ${ }^{12-13 .}$ Este foi escolhido pois é de fácil aplicação e permite avaliar a adesão medicamentosa em três domínios: regime, crenças e recordação. Estes domínios foram analisados pelo escore total de pontos atribuídos as respostas, sendo que todas negativas indicavam adesão ao tratamento e uma ou mais respostas positivas, sugeriram provável não adesão.

Os dados foram tabulados no Microsoft Exce ${ }^{\circledR}$ e analisados por meio de estatística descritiva, com auxílio de uma profissional de estatística. Os resultados foram apresentados em tabelas.

O projeto de pesquisa foi aprovado pelo Comitê de Ética do Setor de Ciências da Saúde da Universidade Federal do Paraná sob CAAE: 07444512.0.0000.0102, parecer 220.068 e respeita a resolução 466/2012. 


\section{Resultados e Discussão}

Os resultados do estudo demonstram que 114 (78,0\%) pacientes eram do sexo feminino, com idade média de 53 anos, variando de 33 a 60 anos e 46 (31,5\%) tinham como profissão atividades do lar, 50 (34,2\%), estudaram durante 0 ensino fundamental de primeira a quarta série e a renda média mensal foi de 934,59 reais. Quanto ao estado conjugal 82 $(56,1 \%)$ eram casados (Tabela 1$)$.

Tabela 1 - Distribuição dos participantes segundo variáveis sociodemográficas. Curitiba - PR, 2015.

\begin{tabular}{|c|c|c|}
\hline Variáveis & $\mathbf{n}$ & $\%$ \\
\hline \multicolumn{3}{|l|}{ Sexo } \\
\hline Masculino & 32 & 21,9 \\
\hline Feminino & 144 & 78,0 \\
\hline \multicolumn{3}{|l|}{ Escolaridade } \\
\hline $7^{\circ}$ a $4^{\circ}$ série & 50 & 34,2 \\
\hline $5^{\circ}$ a $8^{\circ}$ série & 42 & 28,7 \\
\hline $2^{\circ}$ grau & 41 & 28,1 \\
\hline Ensino superior & 13 & 8,9 \\
\hline \multicolumn{3}{|l|}{ Profissão } \\
\hline Do lar & 46 & 31,5 \\
\hline Doméstica/diarista & 10 & 6,8 \\
\hline Autônomo & 12 & 8,2 \\
\hline Aposentado & 15 & 10,2 \\
\hline Pensionista & 8 & 5,4 \\
\hline Encostado & 8 & 5,4 \\
\hline Outros & 47 & 31,5 \\
\hline \multicolumn{3}{|l|}{ Estado conjugal } \\
\hline Solteiro & 19 & 13,0 \\
\hline Casado & 82 & 56,1 \\
\hline Divorciado/separado & 14 & 9,5 \\
\hline Viúvo & 16 & 10,9 \\
\hline Amasiado & 15 & 10,2 \\
\hline
\end{tabular}

A avaliação do Índice de Massa Corporal (IMC), baseados nos valores recomendados pelas Diretrizes brasileiras de obesidade ${ }^{14}$ demonstrou que $80(55,0 \%)$ indivíduos eram obesos. Quanto aos antecedentes familiares $136(93,1 \%)$ referiram possuir pelo menos um antecedente com HAS (Tabela 2). 
Tabela 2 - Distribuição dos participantes de acordo com as variáveis obesidade, antecedentes e doenças familiares, Curitiba-PR, 2015.

\begin{tabular}{|c|c|c|}
\hline Variáveis & $\mathbf{n}$ & $\%$ \\
\hline \multicolumn{3}{|l|}{ Índice de Massa Corporal } \\
\hline Baixo peso ( <18,5kg/m²) & 1 & 0,6 \\
\hline Sobrepeso ( $\geq 25 \mathrm{~kg} / \mathrm{m}^{2} \mathrm{e}<30 \mathrm{~kg} / \mathrm{m}^{2}$ ) & 38 & 26,1 \\
\hline Obesidade $1\left(30,0 \mathrm{~kg} / \mathrm{m}^{2}\right.$ a $\left.34,9 \mathrm{~kg} / \mathrm{m}^{2}\right)$ & 54 & 37,0 \\
\hline Obesidade $2\left(35,0 \mathrm{~kg} / \mathrm{m}^{2}\right.$ a $\left.39,9 \mathrm{~kg} / \mathrm{m}^{2}\right)$ & 15 & 10,3 \\
\hline Obesidade $3\left(\geq 40 \mathrm{~kg} / \mathrm{m}^{2}\right.$ ) & 11 & 7,5 \\
\hline \multicolumn{3}{|l|}{ Antecedentes com HAS } \\
\hline Sim & 136 & 93,1 \\
\hline Não & 10 & 6,9 \\
\hline \multicolumn{3}{|c|}{ Principais doenças familiares e complicações da HAS } \\
\hline Hipertensão arterial sistêmica & 122 & 83,5 \\
\hline Diabetes mellitus & 70 & 47,9 \\
\hline Infarto agudo do miocárdio & 43 & 29,4 \\
\hline Acidente vascular cerebral & 22 & 15,0 \\
\hline Insuficiência renal crônica & 1 & 0,6 \\
\hline
\end{tabular}

Em relação ao tempo de diagnóstico de HAS 50 (34,3\%) possuíam a doença há mais de um ano e menos de cinco e $126(86,4 \%)$ não possuíam complicações (Tabela 3).

Tabela 3 - Distribuição dos participantes segundo as variáveis tempo de diagnóstico e comorbidades. Curitiba- PR, 2015.

\begin{tabular}{c|c|c}
\hline Tempo de diagnóstico & $\mathbf{n}$ & \% \\
\hline 1 a 5 anos & 50 & 34,3 \\
\hline 6 a 10 anos & 37 & 25,3 \\
\hline 10 a 20 anos & 40 & 27,3 \\
\hline Mais de 20 anos & 19 & 13,1 \\
\hline Complicações & 11 & 7,5 \\
\hline Infarto agudo do miocárdio & 6 & 4,1 \\
\hline Acidente vascular cerebral & 1 & 0,7 \\
\hline Insuficiência renal crônica & 2 & 1,3 \\
\hline Retinopatia & 126 & 86,4 \\
\hline Sem complicações &
\end{tabular}


Em relação à classificação de risco da Unidade Básica de Saúde, 67 (45,9\%) participantes foram classificados no estrato de baixo risco para desenvolverem complicações, $44(30,1 \%)$ como médio risco, $28(19,1 \%)$ no alto risco e $7(4,8 \%)$ no muito alto risco.

Ao avaliar o domínio Regime, percebeu-se que mesmo sendo uma amostra de aderentes $5,4 \%$ relataram falhas de dias ou doses e 6,9\% não lembraram de listar espontaneamente todos os medicamentos prescritos.

No domínio Crenças, 145 (99,3\%) acreditam que as medicações trazem benefícios. No domínio Recordação 53 (36,3\%) fazem uso de esquemas com poucas dosagens e dois $(1,4 \%)$ tem facilidade de manusear as embalagens (Tabela4).

Tabela 4 - Distribuição dos participantes segundo o regime medicamentoso nos domínios regime, crenças e recordação. Curitiba-PR, 2015.

\begin{tabular}{|c|c|c|c|c|}
\hline Domínio regime & $\operatorname{sim}$ & $\%$ & Não & $\%$ \\
\hline $\begin{array}{l}\text { Lembraram de listar espontaneamente } \\
\text { todos os medicamentos prescritos }\end{array}$ & 136 & 93,1 & 10 & 6,9 \\
\hline $\begin{array}{c}\text { Relataram problemas na continuidade } \\
\text { da terapêutica devido a atrasos na } \\
\text { dispensação da medicação }\end{array}$ & - & - & 146 & 100 \\
\hline Relataram falhas de dias ou doses & 8 & 5,4 & 138 & 94,6 \\
\hline $\begin{array}{l}\text { Relataram redução ou omissão de doses } \\
\text { de algum medicamento }\end{array}$ & - & - & 146 & 100 \\
\hline $\begin{array}{l}\text { Fez uso de alguma dose extra da } \\
\text { medicação a mais que o prescrito }\end{array}$ & - & - & 146 & 100 \\
\hline \multicolumn{5}{|l|}{ Domínio crenças } \\
\hline $\begin{array}{l}\text { Referiram problemas causados pelas } \\
\text { medicações anti-hipertensivas }\end{array}$ & 1 & 0,7 & 145 & 99,3 \\
\hline $\begin{array}{l}\text { Responderam que alguma medicação } \\
\text { não funciona bem ou que "não sabia" }\end{array}$ & - & - & 146 & 100 \\
\hline \multicolumn{5}{|l|}{ Domínio Recordação } \\
\hline $\begin{array}{l}\text { Recebem um esquema de múltiplas } \\
\text { doses de medicamentos ( } 2 \text { vezes ou } \\
\text { mais/dia) }\end{array}$ & 93 & 63,7 & 53 & 36,3 \\
\hline $\begin{array}{l}\text { Relataram alguma dificuldade com } \\
\text { os medicamentos (abrir ou fechar a } \\
\text { embalagem, ler o que está escrito } \\
\text { na embalagem, lembrar de tomar } \\
\text { todas as doses do medicamento, } \\
\text { conseguir o medicamento, tomar vários } \\
\text { medicamentos ao mesmo tempo) }\end{array}$ & 144 & 98,6 & 2 & 1,4 \\
\hline
\end{tabular}

Mediante análise dos dados verificou-se a predominância de indivíduos do sexo feminino e casados, o que corrobora com o resultado de um estudo quantitativo exploratório, realizado em Maringá, Paraná com 150 hipertensos no qual $102(68 \%)$ eram do sexo feminino e casados ${ }^{15}$. A incidência da HAS em pessoas do sexo feminino também foi observada em outros estudos $1,8,10,15$.

A escolaridade de 50 participantes (34,2\%) da amostra foi de primeira a quarta série e a renda média foi de 724,00 reais, dados que se assemelham aos achados de um estudo realizado com 69 indivíduos da Unidade de Clínica Médica do Hospital das Clínicas da Faculdade de Medicina de Ribeirão Preto, cuja escolaridade predominante foi de primeira a quarta série $(63,8 \%)$ e a renda de 880,00 a 1760,00 reais $(37,7 \%)^{16}$. 
Um estudo descritivo que verificou os facilitadores e dificultadores da adesão a terapêutica medicamentosa de 69 pacientes com HAS, percebeu que os participantes que afirmaram receber mais de um salário mínimo possuíam maiores índices de aquisição das medicações anti-hipertensivas e aqueles com renda familiar de até três salários mínimos, obteve maior número de faltas às consultas médicas, quando comparados aos indivíduos com renda familiar mensal superior $^{16}$. Estes dados contrariam os resultados desta pesquisa, tendo em vista que mesmo em condições econômicas e de escolaridade baixas, os participantes adquiriam as medicações na unidade de saúde.

Ao avaliar o IMC, 80 (54,8\%) indivíduos eram obesos, tal característica vai ao encontro da literatura que afirma que esse é um dos fatores predisponentes para a elevação dos níveis da pressão arterial e responsável por aumentar os riscos para 0 aparecimento de complicações ${ }^{10}$.

$\mathrm{O}$ aumento do IMC em $2,4 \mathrm{~kg} / \mathrm{m}^{2}$ para adultos aumenta a chance de desenvolvimento da HAS, a qual tem relação positiva com a obesidade central ${ }^{2}$. Ao investigarem os principais fatores de risco cardiovascular em Portugal, constataram que 19.856 (51,6\%) participantes estava com obesidade ou excesso de peso e observou-se graus de hipertensão mais severos em pessoas obesas mórbidas (IMC $\geq 40 \mathrm{~kg} / \mathrm{m}^{2}$ ) e que não praticavam exercícios físicos regularmente ${ }^{17}$.

O tempo diagnóstico predominante dos entrevistados variou entre um a cinco anos, o que coincide com os resultados de um estudo realizado em 2011 no interior paulista, no qual 48,6\% da amostra tinham este tempo de diagnóstico ${ }^{8}$. Porém, se somado à quantidade de pacientes com tempo de diagnóstico acima de seis anos temos aproximadamente $70 \%$ da amostra de aderentes, o que contrapõe a literatura que afirma que quanto maior o tempo de diagnóstico maiores são as taxas de abandono da terapêutica².

Nesta pesquisa, $126(86,3 \%)$ participantes não apresentaram complicações cardiovasculares e possuíam baixo risco para desenvolvimento dessas. Visto que se trata de uma amostra de aderentes, tal achado corrobora com a literatura a qual afirma que a adesão ao tratamento está relacionada a redução das complicações da HAS, porém, cerca de $50 \%$ dos hipertensos não mantêm-se aderentes ao tratamento-18.

O domínio de crenças diz respeito à presença ou não de medicamentos que não funcionam bem, ou causam algum problema para o paciente, e ao analisar este domínio percebeu-se que 99,3\% dos entrevistados não referiram quaisquer problemas causados pelas medicações anti-hipertensivas, o que é apontado como fator importante para que ocorra a adesão, pois quando esses medicamentos causam efeitos colaterais os indivíduos tendem a não aderir à terapia. As crenças dos indivíduos sobre a eficácia do tratamento e sobre o conhecimento da doença são facilitadoras da adesão, pois motivam a continuidade do esquema de doses prescritas ${ }^{19}$.

Alguns motivos relacionados às crenças, recordação e regime percebidos nesta amostra, que podem estar relacionados com a adesão a terapêutica referem-se à ausência de dificuldades encontradas com a dispensação da medicação e problemas causados pelas medicações anti-hipertensivas, além dos pacientes recordarem de fazer uso das medicações todos os dias e acreditarem que estas funcionam bem, fatores esses que podem facilitar a continuidade da terapêutica.

Uma limitação encontrada ao realizar esta pesquisa, e que pode ter influenciado na predominância do sexo feminino, foi o local e horário de coleta de dados, pois esta foi realizada em dias úteis, no período da tarde e no domicílio dos entrevistados, período em que e a maior parte da população masculina trabalha. Tal achado também está relacionado com o resultado predominante para ocupação com atividades do lar o que vai ao encontro da literatura que afirma o fato de que mulheres são na maioria donas de casa ou aposentadas e consequentemente possuem maior disponibilidade de horários ${ }^{20}$.

\section{Considerações Finais}

Por meio desta pesquisa foi possível perceber alguns motivos que facilitam a adesão ao tratamento de pessoas com hipertensão, como a lembrança do nome dos medicamentos espontaneamente, ingesta correta das doses prescritas, ausência de problemas causados pelas medicações anti-hipertensivas ou de dificuldades para dispensação da medicação pelos serviços de saúde. Outro fator percebido foi a crença dos indivíduos sobre a eficácia do tratamento e os benefícios que a adesão pode acarretar em suas vidas.

Os fatores listados permitem que os profissionais da saúde conheçam os pacientes, os fatores intervenientes para que eles adiram ao tratamento e dessa maneira, podem utilizar essas informações para o planejamento do cuidado e como artifício de educação em saúde individualizada a fim de traçar estratégias eficazes que possam facilitar a adesão à terapêutica. 


\section{Agradecimentos}

Esta pesquisa recebeu financiamento da FundaçãoAraucária do Paraná e do Conselho Nacional de Desenvolvimento Científico e Tecnológico (CNPQ) pelo Edital Universal.

\section{Referências}

1. Brasil. Ministério da Saúde. Secretaria de Atenção à Saúde. Departamento de Atenção Básica. Estratégias para o cuidado da pessoa com doença crônica. Hipertensão arterial sistêmica. Brasília; 2013.

2. Sociedade Brasileira de Cardiologia; Sociedade Brasileira de Hipertensão; Sociedade Brasileira de Nefrologia. VI Diretrizes brasileiras de hipertensão arterial. São Paulo (SP); 2010.

3. Ferreira FM, Cruz MJB, Santos DF, Linhares MP, Andrade RA. Fatores relacionados à adesão farmacoterapêutica de pacientes hipertensos acolhidos na estratégia de saúde na família. Rev APS [Internet]. 2013 [acesso 2016 jan 10];16(3):258-68.Disponível em: http://aps.ufjf.emnuvens.com.br/aps/article/view/1782/743.

4. Santos ZMSA, Marques ACT, Carvalho YP. Fatores relacionados à adesão ao tratamento do usuário hipertenso. Rev bras promoç saúde [internet]. 2013 [acesso 2015 nov 12];26(2):298-306. Disponível em: http://www.redalyc.org/articulo. oa?id=40828920019.

5. Buitrago F. Adherencia terapéutica. ¡Qué difícil es cumplir! Rev aten prim [internet]. 2011 [acesso 2016 jan 20];46(7):343-4. Disponível em: http://www.elsevier.pt/pt/revistas/-lartigo/adherencia-terapeutica-que-dificil-es-cumplir-90023817.

6. Araujo MFM, Gonçalves TC, Damasceno MMC, Caetano JA. Adesão de diabéticos ao tratamento medicamentoso. Esc Anna Nery Rev Enferm [internet]. 2010 [acesso 2015 jul 14];14(2):361-7. Disponível em: www.scielo.br/pdf/ean/v14n2/20.pdf.

7. Cavalari E, Nogueira MS, Fava SMCL, Cesarino CB, Martin JFV. Adesão ao tratamento: estudo entre portadores de hipertensão arterial sistêmica em seguimento ambulatorial. Rev enferm UERJ [internet]. 2012 [acesso 2015 dez 08];20(1):67-72. Disponível em: http://www.facenf.uerj.br/v20n1/v20n1a12.pdf.

8. Alves BA, Calixto AATF. Aspectos determinantes da adesão ao tratamento de hipertensão e diabetes em uma unidade básica de saúde do interior paulista. J Health Sci Inst [internet]. 2012 [acesso 2015 dez 09];30(3):255-60. Disponível em:http://www.unip.br/comunicacao/publicacoes/ics/edicoes/2012/03_jul- set/V30_n3_2012_p255a260.pdf.

9. Reiners AAO, Seabra FMF, Azevedo RCS, Sudré MRS, Duarte SJH. Adesão ao tratamento de hipertensos da atenção básica. Rev Ciên Cuid Saúde [internet]. 2012 [acesso 2015 dez 08];11(3):581-87. Disponível em: http://eduem.uem.br/ ojs/index.php/CiencCuidSaude/article/viewFile/16511/pdf.

10. Girotto E, Andrade SM, Cabrera MAS, Matsuo T. Adesão ao tratamento farmacológico e não farmacológico e fatores associados na atenção primária da hipertensão arterial. Ciênc saúde coletiva [internet]. 2013 [acesso 2016 jan 05]; 18(6):1763-72. Disponível em: http://www.scielo.br/scielo.php?script=sci_arttext\&pid=S1413-81232013000600027\&lng $=$ pt\&nrm=iso\&tlng=pt.

11. Folstein M. Mini-mental and son. Int J Geriatr Psychiatry.England;1998;13:290-94.

12. Svarstad BL, Chewning BA, Sleath BL, Claesson B. The brief medication questionnaire: A tool for screening patient adherence and barriers to adherence. Patient Educ Couns [Internet]. 1999 [cited 2015 oct 27];37:113-24. Disponível em: http://www.sciencedirect.com/science/article/pii/.

13. Ben AJ, Neumann CR, Mengue SS. Teste de Morisky-Green e Brief Medication Questionnaire para avaliar adesão a medicamentos. Rev. Saúde Pública [internet]. 2012 [acesso 2015 set 08];46(2):279-89. Disponível em: http://www.scielo. $\mathrm{br} / \mathrm{pdf} / \mathrm{rsp} / \mathrm{v} 46 \mathrm{n} 2 / 3357 . \mathrm{pdf}$.

14. Associação Brasileira para o Estudo da Obesidade e da Síndrome Metabólica. Diretrizes brasileiras de obesidade Itapevi (SP); 2009.

15. Demoner MS, Ramos ERP, Pereira ER. Fatores associados à adesão ao tratamento anti-hipertensivo em unidade básica de saúde. Acta paul enferm [internet]. 2012 [acesso 2015 dez 22];25(1):27-34. Disponível em:

http://www.scielo.br/pdf/ape/v25nspe1/pt_05.pdf.

16. Godoy $A C Q$, Veiga EV. Fatores que interferem na adesão terapêutica medicamentosa em hipertensos. Einstein [internet]. 2013 [acesso out 2011];11(3):331-7. Disponível em: http://www.scielo.br/pdf/eins/v11n3/a12v11n3.pdf. 
17. Perdigão C, Rocha E, Duarte JS, Santos A, Macedo A. Prevalência, caracterização e distribuição dos principais factores de risco cardiovascular em Portugal. Uma análise do estudo Amália. Rev Port Cardiol. [internet] 2011 [acesso em 2016 fev 10];30(4):393-432. Disponível em: http://repositorio.ul.pt/handle/10451/5699.

18. Araújo GBS, Garcia TR. Adesão ao tratamento anti-hipertensivo: uma análise conceitual. Rev eletrônica enferm [internet]. 2006 [acesso 2015 nov 14];8(2):259-72. Disponível em: http://www.revistas.ufg.br/index.php/fen/article/view/7041.

19. Soares MM, Silva LOL, Dias CA, Rodrigues SM, Machado CJ. Adesão do idoso ao tratamento da hipertensão arterial sistêmica: revisão integrativa. Cogitare enferm [internet]. 2012 [acesso 2015 jul 14];17(1):144-50. Disponível em: http:// ojs.c3sl.ufpr.br/ojs/index.php/cogitare/article/viewFile/26389/17582.

20. Alves BHS, Prado MA, Góes NC, Beccaria LM, Cesarino CB. Caracterização de usuários hipertensos e adesão ao tratamento em unidade de saúde da família Cogitare enferm [internet]. 2012 [acesso 2015 jul 14];17(1):91-8. Disponível em: http://ojs.c3sl.ufpr.br/ojs/index.php/cogitare/article/view/26380/17573.

\section{Maria de Fátima Mantovani}

Endereço para correspondência - Universidade Federal do Paraná, Setor de Ciências da Saúde, Departamento de Enfermagem, Bloco Didático II - Campus Botânico: Av. Lothário Meissner, 632 Jardim Botânico, CEP: 80210-170, Curitiba, PR, Brasil

E-mail: mfatimamantovani@ufpr.br Lattes: http://lattes.cnpq.br/8732441427702807

Ângela Taís Mattei - angela-mattei@hotmail.com Evelyn Nunes da Rocha - evelyn.rocha24@gmail.com Carina Bortolato Major - cabortolato@uenp.edu.br

Elis Martins Ulbrich - lilaulbrich@yahoo.com.br Karin Cristina Barboza - karin.cristina.barboza@gmail.com

\section{Enviado em 16 de março de 2016. Aceito em 09 de maio de 2016.}

